\title{
Collagen IV: a critical new starting point for engineering upper airways
}

\author{
Martina M. De Santis (1) ${ }^{1,2,3}$ and Darcy E. Wagner (1) ${ }^{1,2,3}$
}

Affiliations: ${ }^{1}$ Lung Bioengineering and Regeneration, Dept of Experimental Medical Sciences, Faculty of Medicine, Lund University, Lund, Sweden. ${ }^{2}$ Wallenberg Molecular Medicine Center, Faculty of Medicine, Lund University, Lund, Sweden. ${ }^{3}$ Lund Stem Cell Center, Faculty of Medicine, Lund University, Lund, Sweden.

Correspondence: Darcy E. Wagner, Lund University, Dept of Experimental Medical Sciences, B10 BMC Lund, Sweden. E-mail: darcy.wagnerlamed.lu.se

@ERSpublications

Collagen IV and integrin alpha 2 engagement is critical for forming differentiated airway epithelium capable of mucociliary clearance in vitro, but pre-vascularised scaffolds transplanted under immunosuppresion fail to maintain differentiated epithelium https://bit.ly/2wT9XK5

Cite this article as: De Santis MM, Wagner DE. Collagen IV: a critical new starting point for engineering upper airways. Eur Respir J 2020; 55: 2001130 [https://doi.org/10.1183/13993003.01130-2020].

Half a century has passed since the discovery of collagen IV in 1966 by KeFALIDEs [1]. Collagen IV is the main collagen component of the basement membrane in many organs, including the lung and trachea. It is a network-forming collagen that underlies epithelial and endothelial cells and serves a crucial role in forming barriers between tissue compartments [2,3]. Mature collagen IV is a heterotrimer comprised from a combination of two or three of the six known collagen IV alpha chains which are increasingly recognised as being tissue- and organ-specific. Integrin $\alpha 2$ is a known cellular receptor for attachment to collagen IV and through interactions with different binding partners, influences cell adhesion, migration and polarity [4].

In this issue of the European Respiratory Journal, HAMILTON et al. [5] investigate the influence of collagen IV, integrin $\alpha 2$ and other extracellular matrix (ECM) components and integrins on airway epithelial cell adherence, proliferation and mucociliary function for engineering upper airways. They observe that in vitro, primary human airway epithelial cells adhere most readily to collagen IV via integrin $\alpha 2$ but that collagen IV is lost following tracheal decellularisation. They then test the novel concept of using a collagen IV-rich substrate derived from decellularised dermis as the substrate for initial cellular attachment and test whether it can support the formation and differentiation of primary basal epithelial cells into a respiratory mucosal layer with mucociliary function. They then attach this layer to a pre-vascularised decellularised trachea and test the feasibility of this two-step approach using an air-liquid interface culture system, a chick chorioallantoic membrane (CAM) assay, an ectopic rabbit airway transplantation model, and subcutaneous transplants in NOD SCID mice. Importantly, they tested their constructs in animal models which utilised clinically relevant immunosuppression regimes, such as tacrolimus, which is commonly used in allogeneic organ transplants and dexamethasone, an anti-inflammatory drug. The preliminary in vitro data is promising and they demonstrate that the delivery of these complex scaffolds is feasible in vivo and that epithelium is retained in the short-term. Ultimately, however, the respiratory epithelium does not survive long-term using this approach.

Both biologically derived and manufactured scaffolds seeded with cells and grown ex vivo have been explored for generating airways or pulmonary tissue for transplantation [6]. While clinical transplantation of de- and re-cellularised airways as well as synthetic airways has been performed, clinical outcomes have 
been poor with low patient survival [7]. Recently, substantial insights into the significant clinical challenges facing the field of bioengineering upper airways have been transparently highlighted through detailed clinical case reports [8]. Development of pre-clinical assays and in vivo models which can be used to predict and better model clinical outcomes has been known to be an important but challenging area in this field [9].

Despite its controversy, the field of airway tissue engineering has made major achievements in the last few years towards developing clinical-grade methods for generating airways for transplantation. Methods to isolate and scale up the required number of cells needed for covering the surface area of bioengineered upper airways for transplantation have been developed [10], as well as studies which describe methods to promote engraftment of epithelial progenitor cells [11]. Additionally, the importance of an intact vascular network for tracheal and large airway graft survival is well-recognised [12] and there have been innovative techniques to encourage vascularisation $[13,14]$. While multiple protocols have been described for decellularising large airways and lung tissue, the functional role of specific ECM components, including proteins, proteoglycans and glycosaminoglycans, is not fully understood [15]. The optimal starting ECM composition for generating functional large airways or lung tissue is not yet known, but recent work comparing scaffolds derived from healthy animals or humans versus those from aged or diseased sources indicate that the ECM composition of the bioengineered airway and lung will play a significant role in initial cellular behaviour and the clinical outcome. Aged and diseased lungs have been found to have different protein profiles compared to healthy lungs and significantly regulate cellular behaviour $[6,16$, 17]. The study by Hamilton et al. [5], particularly the CAM assay and in vivo animal models, could be used to help assess the best combination of ECM components that are required to engineer a respiratory mucosal layer that can survive and function long term in vivo.

As shown by HAMILTON et al. [5], collagen IV is a promising candidate for bioengineering respiratory mucosal layers by providing initial support for epithelial cell attachment and differentiation, but it is not clear in what amounts and in which combination this ECM component is optimal for bioengineering respiratory mucosal layers. Several previous studies have characterised the effect of detergents used for decellularisation on retention of collagen IV in the lung and in small airways $[18,19]$. The majority of studies have assessed the presence of collagen IV abundance via peptide identification using mass spectrometry proteomics [20], which can be difficult to interpret given the challenges with solubilising collagens. Some reports indicate that there is a general loss of collagen IV with decellularisation using detergents [21], while other studies have found that most of the detergents commonly used for generating acellular lung and airway scaffolds differentially induce degradation and changes in collagen IV solubility [18]. However, most of the previous studies focused on the retention and integrity of collagen IV and other proteins at the bulk tissue level. HamiLton et al. [5] find that their protocol for producing acellular tracheal grafts from rabbits meets the criteria for a decellularised construct with regard to cellular removal, maintenance of the major architecture and low DNA content, but find a concomitant and noticeable loss of collagen IV. Subsequently, they observe low adherence of human airway epithelial cells to these acellular tracheas. The authors overcome this by using a collagen IV-rich substrate derived from acellular human dermis produced using a detergent-free protocol. Due to the fact that acellular dermis does not have the mechanical strength to be used in airway tissue engineering, they attach this substrate to the acellular trachea. While innovative, this two-step approach may introduce other major problems, such as delamination, if the two layers are not sufficiently adherent to one another. Identification and validation of decellularisation methods which preserve the right ECM proteins in the right anatomical location are needed for informing future decellularisation and airway engineering strategies. Alternatively, methods to add specific ECM components back to acellular constructs could be an interesting approach to explore in subsequent studies.

In addition to decellularised ECM, synthetic, natural or hybrid materials which are designed for airway tissue engineering still remain a viable option but need extensive optimisation [6]. The work by HamiLton et al. [5] indicates that scaffolds with collagen IV or peptide sequences which can promote engagement with specific integrins, such as integrin $\alpha 2$, are promising options to explore in the future. These scaffolds could be further engineered to include other signalling cues, including the release of growth factors, or regional control over mechanical properties to mimic those needed for engineering a respiratory mucosal layer. There are many open questions left to explore such as, what other ECM components are required, in which amounts, which type of signalling cues and what the required mechanical properties are.

Another key issue raised by HAMILTON et al. [5] is the transplantation of a differentiated cell layer versus a basal cell-only layer. Transplanting differentiated airway epithelial cells could restore tissue function more rapidly, but differentiated epithelial cells were lost over time when transplanted ectopically into the backs of NOD SCID mice [5]. This may be due to a lack of polarity signals available to the epithelial cells in the ectopic position. Alternatively, recent work has shown that loss of basal stem cell subsets (submucosal 
gland cells $(\mathrm{Krt5}+/ \mathrm{Krt14+})$ and basal stem cells $(\mathrm{p} 63+/ \mathrm{Krt5}+))$ correlate with pulmonary allograft failure, clinically as well as in a ferret model of transplantation, which also uses immunosuppressive regimes [22]. Characterisation of the immune response, including the role of infiltrating cells on the maintenance of mature airway epithelial cells and basal stem cell subsets, is an important aspect to consider for transplant biology, including for engineered tissues. In addition, future studies which transplant engineered airways orthopically will be critical to identify whether differentiated airway epithelial cells can retain their differentiated phenotype. Other cues present in the orthotopic position, such as shear stress induced by the movement of air or distal to proximal mucociliary clearance, may help support retention of a multicellular epithelium. On the other hand, transplanting undifferentiated basal stem cells could be simpler and faster for transplantation as the cells do not need to be differentiated in vitro, but it is unclear whether the transplanted basal stem cells will have the necessary cues to differentiate into a multilayered epithelium over time in vivo [11]. One potential possibility to overcome this challenge could be to engineer a scaffold which includes spatiotemporal signalling cues to direct the differentiation of the basal stem cells or, in the case of a transplanted differentiated epithelium, work to maintain a multi-layered epithelium over time.

In addition to defining critical components in the initial scaffold, it is known that an intact vascular network is critical for long term graft function [12-14]. In order to work towards this, HAMiLTON et al. [5] first generated a pre-vascularised tracheal layer by transplanting acellular rabbit tracheas in a lateral thoracic muscle flap for 4 weeks. They then grafted the engineered, differentiated respiratory mucosal layer onto this pre-vascularised tracheal layer, aiming for faster graft integration and function. While they observed histological evidence of vascular ingrowth to the respiratory mucosal layer after five days, the epithelial layer was lost either due to cell death or weak integration of the two layers [5]. Future studies which study methods to chemically attach or bond the two tissue layers, or attachment of a thinner epithelial graft layer could help overcome this potential hurdle.

In conclusion, HAMILTON et al. [5] have moved the airway tissue engineering field a few steps closer to clinical reality by identifying collagen IV as a key starting point in the scaffold for epithelial cell attachment. They further establish critical methods to ectopically transplant differentiated airway epithelium grafted on a pre-vascularised airway scaffold into rabbits. Importantly, they have established a solid pre-clinical workflow using an ex vivo CAM assay and in vivo animal models which can be used to assess potential tissue engineered upper airway respiratory grafts. A number of important points to explore in the future remain, such as surgical techniques, including stenting methods, and the role of immunosuppressive regimes on epithelial cell maintenance and differentiation. The work by HaмiLTON et al. [5] gives a new perspective on identifying critical starting components for engineering functional airways, but highlights the need for further studies to gain knowledge on how to maintain mature epithelium after transplantation to bring the field closer to establishing transplantable, bioengineered airways which can function over time.

Conflict of interest: D.E. Wagner reports grants from Knut och Alice Wallenbergs Stiftelse, during the conduct of the study; personal fees from Boehringer Ingelheim, outside the submitted work; and has a patent WO2014169111A1 pending. M.M. De Santis has nothing to disclose.

Support statement: The Knut and Alice Wallenberg foundation, the Medical Faculty at Lund University and Region Skåne are acknowledged for generous financial support (D.E. Wagner). Funding information for this article has been deposited with the Crossref Funder Registry.

\section{References}

1 Kefalides NA. A collagen of unusual composition and a glycoprotein isolated from canine glomerular basement membrane. Biochem Biophys Res Commun 1966; 22: 26-32.

2 Khoshnoodi J, Pedchenko V, Hudson BG. Mammalian collagen IV. Microsc Res Tech 2008; 71: 357-370.

3 Sand JMB, Genovese F, Gudmann NS, et al. Chapter 4 - Type IV collagen. In: Karsdal MA, ed. Biochemistry of Collagens, Laminins and Elastin. 2nd Edn. Academic Press, 2019; pp. 37-49.

4 Manninen A. Epithelial polarity - Generating and integrating signals from the ECM with integrins. Exp Cell Res 2015; 334: 337-349.

5 Hamilton NJI, Lee DDH, Gowers KHC, et al. Bioengineered airway epithelial grafts with mucociliary function based on collagen IV- and laminin-containing extracellular matrix scaffolds. Eur Respir J 2020; 55: 1901200.

6 De Santis MM, Bolukbas DA, Lindstedt $S$, et al. How to build a lung: latest advances and emerging themes in lung bioengineering. Eur Respir J 2018; 52: 1601355.

7 Udelsman B, Mathisen DJ, Ott HC. A reassessment of tracheal substitutes-a systematic review. Ann Cardiothorac Surg 2018; 7: 175-182.

8 Elliott MJ, Butler CR, Varanou-Jenkins A, et al. Tracheal replacement therapy with a stem cell-seeded graft: lessons from compassionate use application of a GMP-compliant tissue-engineered medicine. Stem Cells Transl Med 2017; 6: 1458-1464.

9 The Lancet. The final verdict on Paolo Macchiarini: guilty of misconduct. Lancet 2018; 392: 2. 
10 Butler CR, Hynds RE, Gowers KH, et al. Rapid expansion of human epithelial stem cells suitable for airway tissue engineering. Am J Respir Crit Care Med 2016; 194: 156-168.

11 Hamilton NJI, Hynds RE, Gowers KHC, et al. Using a three-dimensional collagen matrix to deliver respiratory progenitor cells to decellularized trachea in vivo. Tissue Eng Part C Methods 2019; 25: 93-102.

12 Chang WG, Niklason LE. A short discourse on vascular tissue engineering. NPJ Regen Med 2017; 2: 7.

13 Delaere P, Vranckx J, Verleden G, et al. Tracheal allotransplantation after withdrawal of immunosuppressive therapy. N Engl J Med 2010; 362: 138-145.

14 Delaere PR, Vranckx JJ, Meulemans J, et al. Learning curve in tracheal allotransplantation. Am J Transplant 2012; 12: $2538-2545$

15 Uhl FE, Zhang F, Pouliot RA, et al. Functional role of glycosaminoglycans in decellularized lung extracellular matrix. Acta Biomater 2020; 102: 231-246.

16 Wagner DE, Bonenfant NR, Parsons CS, et al. Comparative decellularization and recellularization of normal versus emphysematous human lungs. Biomaterials 2014; 35: 3281-3297.

17 Gilpin SE, Wagner DE. Acellular human lung scaffolds to model lung disease and tissue regeneration. Eur Resp Rev 2018; 27: 180021.

18 Wallis JM, Borg ZD, Daly AB, et al. Comparative assessment of detergent-based protocols for mouse lung de-cellularization and re-cellularization. Tissue Eng Part C Methods 2012; 18: 420-432.

19 Gilpin SE, Guyette JP, Gonzalez G, et al. Perfusion decellularization of human and porcine lungs: bringing the matrix to clinical scale. J Heart Lung Transpl 2014; 33: 298-308.

20 O’Neill JD, Anfang R, Anandappa A, et al. Decellularization of human and porcine lung tissues for pulmonary tissue engineering. Ann Thorac Surg 2013; 96: 1046-1056.

21 Hill RC, Calle EA, Dzieciatkowska M, et al. Quantification of extracellular matrix proteins from a rat lung scaffold to provide a molecular readout for tissue engineering. Mol Cell Proteomics 2015; 14: 961-973.

22 Swatek AM, Lynch TJ, Crooke AK, et al. Depletion of airway submucosal glands and TP63(+)KRT5(+) basal cells in obliterative bronchiolitis. Am J Respir Crit Care Med 2018; 197: 1045-1057. 